Supporting Information for

\title{
Scalable mRNA and siRNA Lipid Nanoparticle Production Using a Parallelized Microfluidic Device
}

Sarah J. Shepherd, ${ }^{1}$ Claude C. Warzecha, ${ }^{2}$ Sagar Yadavali, ${ }^{1}$ Rakan El-Mayta, ${ }^{1}$ Mohamad-Gabriel Alahmeh, ${ }^{3}$ Lili Wang, ${ }^{2}$ James M. Wilson, ${ }^{2}$ Drew Weissman, ${ }^{3}$ David Issadore, ${ }^{1,4,5 * \dagger}$ Michael J.

$$
\text { Mitchell } 1^{1,6,7,8,9 * \dagger}
$$

${ }^{1}$ Department of Bioengineering, University of Pennsylvania, Philadelphia, PA 19104 USA.

${ }^{2}$ Gene Therapy Program, Perelman School of Medicine, University of Pennsylvania, Philadelphia, PA 19104 USA.

${ }^{3}$ Department of Medicine, University of Pennsylvania, Philadelphia, PA 19104 USA.

${ }^{4}$ Department of Electrical and Systems Engineering, University of Pennsylvania, Philadelphia, PA 19104 USA.

${ }^{5}$ Department of Chemical and Biomolecular Engineering, University of Pennsylvania, Philadelphia, PA 19104 USA.

${ }^{6}$ Abramson Cancer Center, Perelman School of Medicine, University of Pennsylvania, Philadelphia, PA 19104 USA.

${ }^{7}$ Institute for Immunology, Perelman School of Medicine, University of Pennsylvania, Philadelphia, PA 19104 USA.

${ }^{8}$ Cardiovascular Institute, Perelman School of Medicine, University of Pennsylvania, Philadelphia, PA 19104 USA.

${ }^{9}$ Institute for Regenerative Medicine, Perelman School of Medicine, University of Pennsylvania, Philadelphia, PA 19104 USA.

$\dagger$ These authors contributed equally to this work.

*Corresponding author: Michael J. Mitchell. Email mjmitch@seas.upenn.edu

*Co-corresponding author: David Issadore. Email issadore@seas.upenn.edu

This file contains materials and methods, followed by supplementary figures S1-S6. 


\section{Device design}

\section{Materials and Methods}

The PMD was designed to ensure uniform flow distribution across the device such that the fluidic resistance of each mixing unit is much greater than that of the delivery channels. Using flow resistors to set the device flow resistance, we were able to incorporate a specific number of devices per row $\mathrm{N}_{\text {device }}$ using a published design rule that relates the number of devices allowed per row $\left(\mathrm{N}_{\text {device }}\right)$ to the resistance of delivery channels $\left(\mathrm{R}_{\text {delivery }}\right)$ and resistance of devices: ${ }^{1}$

$$
2 N_{\text {device }}\left(R_{\text {delivery }} / R_{\text {device }}\right)<0.01 \text {. }
$$

The fluidic resistance of the rectangular channels in our device were estimated as $\mathrm{R} \alpha \mu l / w h^{3}$, in the condition where $h<w$, and where $\mu$ is the fluidic viscosity, $l$ is the length, $w$ is the width, and $h$ is the height of the channel. In our design, the maximum number of allowable channels per row was $\mathrm{N}_{\text {device }}=112$, and we conservatively included 32 devices per row. Additionally, our PMD had to satisfy the design constraint to ensure that each row received the same flow rate from the arterial delivery channel-a constraint that is related to the resistance of the supply channel between delivery channel rows $\left(\mathrm{R}_{\text {supply }}\right)$ and the resistance of each row $\left(\mathrm{R}_{\text {row }}\right)$. We approximated the resistance of each row by dividing the resistance of each device by the number of devices per row $\left(\mathrm{R}_{\text {row }}=\mathrm{R}_{\text {device }} / \mathrm{N}_{\text {device}}\right)$ such that each row would be considered $\mathrm{N}_{\text {device }}=32$ mixing channels in parallel. Thus, the design rule for choosing the number of rows was:

$$
2 N_{\text {row }}\left(R_{\text {supply }} / R_{\text {row }}\right)<0.01
$$

By this constraint, in our design we could have a maximum number of rows $\mathrm{N}_{\text {row }}=6$, and we conservatively designed our PMD with 4 delivery channel rows.

\section{Device fabrication}

The fabrication strategy for the PMD used a previously published technique, called double-sided imprinting, ${ }^{2}$ that enables fabrication of 3D structures by combining a multi-layer negative photoresist (SU8) hard master with a PDMS-based soft master. Standard photolithography processes were used to fabricate the multi-layer SU-8 masters, where SU-8 was spin-coated to the desired thicknesses on a silicon wafer (University Wafer), followed by UV exposure on a mask aligner (SUSS MicroTec) for each layer using a $365 \mathrm{~nm}$ long-pass filter. For the PMD (128 mixing channels), five mask layers were designed for the hard master: filters and resistors (layer 1-height $50 \mu \mathrm{m}$ ), mixing channels (layer 2-height $20 \mu \mathrm{m}$ ), herringbones (layer 3-height $25 \mu \mathrm{m}$ ), delivery channels (layer 4 - height $100 \mu \mathrm{m}$ ), supply channels and vias (layer 5-height $200 \mu \mathrm{m}$ ). For the soft master, two mask layers were designed: supply channels (layer 1 - height $350 \mu \mathrm{m}$ ) and filters (layer 2-height $50 \mu \mathrm{m}$ ). For the single row PMD, three mask layers were designed for the hard master: filters, resistors and mixing channels (layer 1-height $70 \mu \mathrm{m}$ ), herringbones (layer 2-height $25 \mu \mathrm{m}$ ), vias and delivery channels (layer 3-height $100 \mu \mathrm{m}$ ). For the single row PMD soft master, two mask layers were designed: delivery channels (layer 1-height $130 \mu \mathrm{m}$ ), and filters (layer 2 - height $70 \mu \mathrm{m}$ ). The PDMS soft masters were prepared by pouring a 10:1 mixture of PDMS elastomer to curing agent (Thermo Fisher) on the Si masters.

To apply the double-sided imprinting technique to fabricate the PMDs, the soft masters must be silanized with trichloro $(1 \mathrm{H}, 1 \mathrm{H}, 2 \mathrm{H}, 2 \mathrm{H}$-perfluorooctyl)silane (MilliporeSigma) following plasma treatment 
to prevent adhesion of the PDMS soft master to the central PDMS piece. PDMS is poured onto the silanized soft and hard masters, and the designs are aligned using support patterns $(2.5 \mathrm{~mm} \times 2.5 \mathrm{~mm}$ for single row PMD; $3 \mathrm{~mm}$ x $3 \mathrm{~mm}$ for PMD) that are incorporated to the design of soft and hard masters. Once alignment is complete, pressure is applied using five $0.5 \mathrm{~kg}$ weights to ensure that the hard master vias maintain contact with the supply channels of the soft master and the PDMS is cured on a $65^{\circ} \mathrm{C}$ hotplate for two hours. The 3D designed PDMS layer is bonded to another piece of PDMS using plasma treatment, input/output holes are punched using a $1 \mathrm{~mm}$ biopsy punch (Thermo Fisher), and the backside of the hole-punched PDMS double-layer device is bonded to a glass slide using plasma treatment. For the single channel device, two mask layers were designed: mixing channels (layer 1 - height $70 \mu \mathrm{m}$ ) and herringbones (layer 2 - height $25 \mu \mathrm{m})$. Devices were fabricated by curing PDMS on the Si master, hole punching with a 1mm biopsy punch (Thermo Fisher) and bonding to a glass slide using plasma treatment.

\section{Experimental setup}

A pressure-driven flow system was used to conduct all experiments for the PMDs. Nitrogen pressure tanks were connected to either $100 \mathrm{~mL}$ or $1 \mathrm{~L}$ Duran pressure plus glass bottles (MilliporeSigma) using PTFE tubing (McMaster-Carr). Pressure regulators (Global Test Supply, LLC) and pressure gauges (Grainger, Inc.) were used to monitor pressures for each inlet, and total volumetric flow rate was measured at the output collection of the device. Microscopy was used to monitor PMD performance and ensure a 3:1 flow rate ratio of nucleic acid input to lipid input. For the single channel device, a Pump 33 DDS (Dual Drive System) (Harvard Apparatus) powered two glass syringes (Hamilton Company) for the nucleic acid and lipid phases at a 3:1 flow rate ratio.

\section{Mixing characterization}

FITC-dextran and rhodamine B isothiocyanate-dextran (MilliporeSigma) were dissolved to $10 \mu \mathrm{M}$ in water. These solutions were flowed through the single channel device, single row PMD, and PMD at a 3:1 flow rate ratio (FITC to rhodamine B) at various flow rates - where the total volumetric flow rate ranged from $24 \mu \mathrm{L} / \mathrm{min}$ to $2400 \mu \mathrm{L} / \mathrm{min}$ per channel. Fluorescence images of the channel were taken at different channel lengths and the intensity profile of each dye across the channel was quantified using Image (NIH) before being exported to MATLAB (MathWorks) for numerical analysis. Fluorescence intensity of FITC and rhodamine $\mathrm{B}$ were processed by subtracting the background, normalizing the intensity, and calculating the area under the curve for the difference of the rhodamine B normalized intensity from the FITC normalized intensity - generating a mixing value that ranged from 1 (not mixed) to 0 (completely mixed). Thus, mixing was plotted as a function of channel length, and values were normalized to a mixing value of 1 at the beginning of the channel ( 0 cycles). The channel length needed for $90 \%$ mixing was calculated based on the intersection of an exponential decay model and a mixing value of 0.1 . Errors reported are the standard error of the exponential decay model.

\section{Lipid nanoparticle (LNP) formulation and characterization}

LNPs were formulated by rapid mixing of an aqueous phase containing RNA and an ethanol phase containing cholesterol and lipids using a microfluidic device or by pipette mixing. The ionizable lipid C12200 was synthesized as previously described. ${ }^{3,4}$ For the in vitro experiments, a formulation of C12-200, 1,2dioleoyl-sn-glycero-3-phosphoethanolamine (DOPE) (Avanti Polar Lipids, Inc.), cholesterol (MilliporeSigma) and 1,2-dimyristoyl-sn-glycero-3-phosphoethanolamine-N-[methoxy(polyethylene glycol)-2000] (PEG2000-PE) (Avanti Polar Lipids, Inc.) were combined at molar ratios of 50\%, 10\%, 
$38.5 \%$, and $1.5 \%$, respectively. For the in vivo siRNA experiments, a formulation of C12-200, 1,2distearoyl-sn-glycero-3-phosphocholine (DSPC), cholesterol, and PEG2000-PE was combined at molar ratios of $50 \%, 10 \%, 38.5 \%$, and $1.5 \%$, respectively. For the in vivo mRNA experiments, a formulation of C12-200, DOPE, cholesterol, and PEG2000-PE were combined at molar ratios of 35\%, 16\%, 46.5\%, and $2.5 \%$, respectively. LNPs formulated by the single row PMD had reagents diluted by $2 \mathrm{x}$ or $3 \mathrm{x}$ to preserve reagents. The C12-200:RNA weight ratio was 10:1 for in vitro siRNA experiments, 5:1 for in vivo siRNA experiments, and 10:1 for in vivo mRNA experiments.

Anti-firefly luciferase siRNA (Dharmacon) was used for in vitro experiments. Anti-Factor VII siRNA (MilliporeSigma) was custom ordered with a sense sequence of 5'-GGAucAucucAAGucuuAcT*T-3' and an anti-sense sequence of 5'-GuAAGAcuuGAGAuGAuccT*T-3', where asterisks indicate phosphorothioate linkages and lowercase nucleotides are 2'-fluoro-modified. ssDNA LNPs were formulated using C12-200, DOPE, cholesterol, and PEG2000-PE at molar ratios of 35\%, 16\%, $46.5 \%$, and $2.5 \%$, respectively with a C12-200:DNA weight ratio of 10:1. ssDNA was purchased from IDT with a sequence of A* $\mathrm{G}^{*} \mathrm{~A}$ *CGTGTGCTCTTCCGATCTGAGGGTACTTNNNNNNNNNNAGATCGGAAGAGCGTCG* $\mathrm{T}^{*} \mathrm{G}^{*} \mathrm{~T}$, where asterisks indicate phosphorothioate linkages and $\mathrm{N}$ indicates randomly generated nucleotides. Firefly luciferase mRNA was synthesized as previously described. ${ }^{5}$

Using the syringe pump system for the single channel device or the pressure-driven flow system for PMDs, a 3:1 flow rate ratio of aqueous to ethanol phases was used for nanoprecipitation of LNPs. Flow rate per channel ranged from $0.06 \mathrm{~mL} / \mathrm{min}$ to $1.26 \mathrm{~mL} / \mathrm{min}$ total volumetric flow rate. LNPs were then dialyzed against $1 \mathrm{X}$ PBS for two hours before filtration by $0.22 \mu \mathrm{m}$ filters. For in vivo experiments, LNPs were concentrated using 50K MWCO centrifugal filters following dialysis (MilliporeSigma). For the pipette mixed LNPs, an electronic pipette (Eppendorf, Thermo Fisher) mixed the reagents at the maximum flow rate. After dialysis in $1 \mathrm{X}$ PBS for two hours, LNPs were filtered by $0.45 \mu \mathrm{m}$ filters. For characterization, LNPs were diluted 1:100 in 1X PBS and dynamic light scattering (DLS) was performed by a Zetasizer Nano (Malvern Instruments) to measure the hydrodynamic diameter (z-average) and polydispersity index (PDI) in triplicate. All sizes reported are intensity weighted averages. Standard deviation of the particle size was calculated by: $\sigma=\sqrt{P D I \cdot \text { diameter }^{2}}$. RNA concentration was quantified by absorbance at A260 using a Tecan NanoQuant Plate (Thermo Fisher). Encapsulation efficiency was calculated by a Quant-iT RiboGreen (Thermo Fisher) assay. Briefly, LNPs were either diluted in TE buffer as a control, or Triton X-100 (MilliporeSigma) to lyse the LNPs and were plated with RNA standards. The RiboGreen reagent was added, and fluorescence was measured by a plate reader. A standard curve was generated to quantify RNA content, and encapsulation efficiency (\%) was calculated as follows: $100 *$ (total RNA - unencapsulated RNA)/(total RNA).

siRNA delivery to HeLa cells in vitro

HeLa cells, stably modified to express firefly luciferase, were cultured in Dulbecco's Modified Eagles Media (DMEM) (Thermo Fisher) supplemented with 10\% fetal bovine serum and $1 \%$ penicillin/streptomycin. The cells were incubated at $37^{\circ} \mathrm{C}$ in $5 \% \mathrm{CO}_{2}$ and plated at a density of 10,000 cells per well (Thermo Fisher). Cells were treated with 1-10 nM siRNA 24 hours after plating, and firefly luciferase expression was measured 24 hours after LNP transfection using a Luciferase Assay System (Promega) according to the manufacturer's protocol. The luminescent signal was normalized to untreated 
cells after subtracting the background (wells without cells with reagents). To assess cytotoxicity, a CellTiter-Glo Luminescent Cell Viability Assay (Promega) was performed according to manufacturer's instructions, and luminescence was normalized to untreated cells after subtracting the background.

\section{In vivo LNP studies}

All animal procedures were performed on male C57B1/6 mice aged 6-8 weeks (The Jackson Laboratory) in accordance with protocols approved by the Institutional Animal Care and Use Committee of the University of Pennsylvania. Mice were administered a single $0.1 \mathrm{~mL}$ intravenous dose of formulated LNPs encapsulating siRNA or mRNA in PBS pH 7.4 (Gibco) via tail vein injection. Plasma was obtained by collecting blood in tubes containing a solution of 3.2\% sodium citrate at a ratio of 9:1 and spun at 2500 $\mathrm{X} \mathrm{g}$ for 10 minutes. Factor VII protein levels were analyzed by a chromogenic enzyme activity assay (Biophen FVII, Aniara) according to the manufacturer's protocol. A standard curve was constructed using commercially available normal mouse plasma (Sigma) and relative Factor VII expression was determined by comparing treated animals to the corresponding pre-treatment sample. Bioluminescence imaging was performed with an IVIS Spectrum imaging system (Caliper Life Sciences). Mice were administered Dluciferin (PerkinElmer) at a dose of $150 \mathrm{mg} / \mathrm{kg}$ by intraperitoneal injection (IP) and anesthetized 5 minutes later by ketamine/xylazine (IP). Image acquisition occurred 20 minutes after D-luciferin administration using an exposure time of 30 seconds with medium binning. Bioluminescence values were quantified by measuring photon flux in the region of interest where bioluminescence signal emanated using the Living IMAGE Software provided by Caliper. At the terminal time point mice were anesthetized by IP injection of ketamine/xylazine and blood was collected via cardiac puncture. Subsequently, mice were euthanized by cervical dislocation and the left lobe of the liver was collected and fixed in $10 \%$ neutral buffered formalin (Fisher Healthcare). Blood collection at all other time points was performed by submandibular bleeding. Hematoxylin and eosin staining were performed on sections from paraffin-embedded liver samples according to standard protocols. Liver function tests were performed by Antech and were compared to previously published reference ranges. ${ }^{6}$

\section{Statistical analysis}

Statistical tests were performed in Graphpad Prism 8. Data were plotted as mean \pm standard deviation unless otherwise specified. Differences between groups were determined by unpaired t test to an appropriate control or one-way analysis of variance (ANOVA) (specified in figure legends). 


\section{Supplementary Figures}
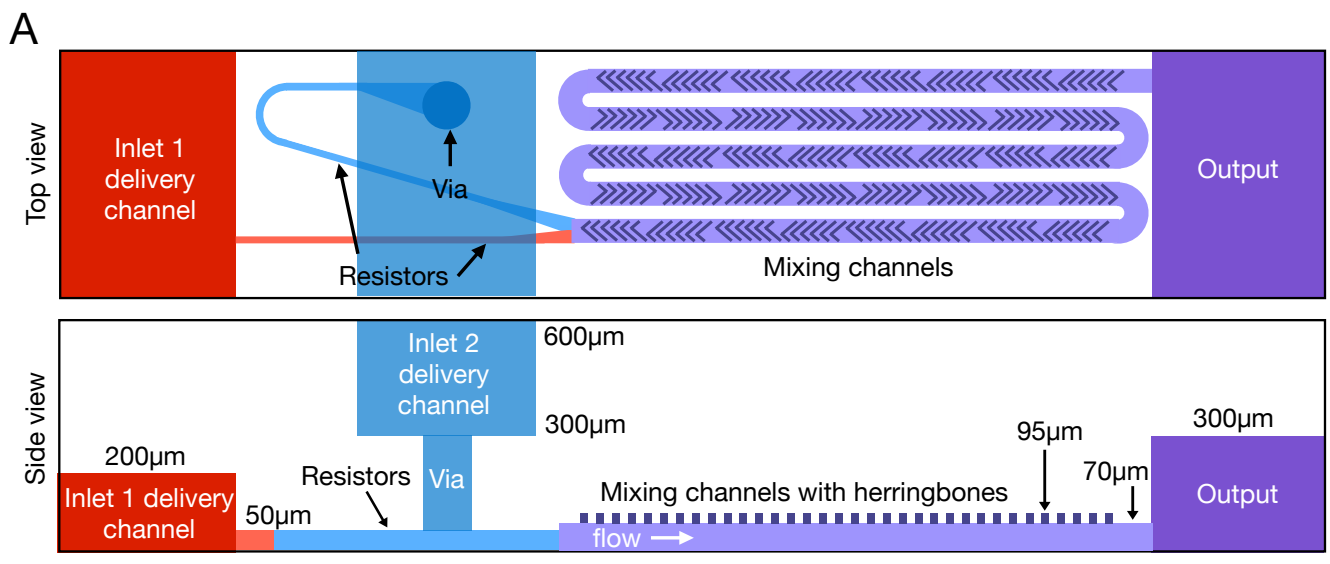

B

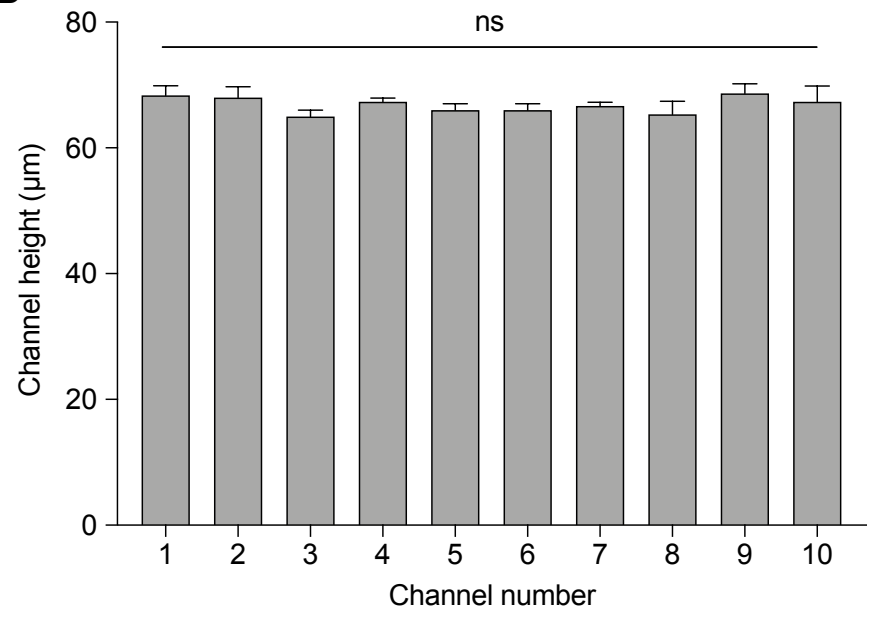

C

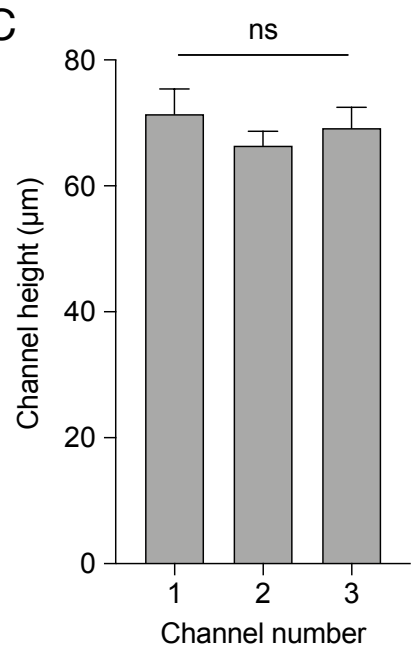

Figure S1. Design of PMD and validation of uniform fabrication.

(A) Schematic for each mixing unit in the PMD, highlighting the heights for each layer in side view. Direction of flow is indicated by white arrows. Schematic is not to scale. (B-C) Validation of uniform channel heights across the single row PMD (B) and PMD (C). Device was cut vertically with a razor blade and channel height was quantified by microscopy. Desired height was $70 \mu \mathrm{m} . \mathrm{n}=3$. Samples were compared by one-way ANOVA. ns: $\mathrm{p}>0.05$. 

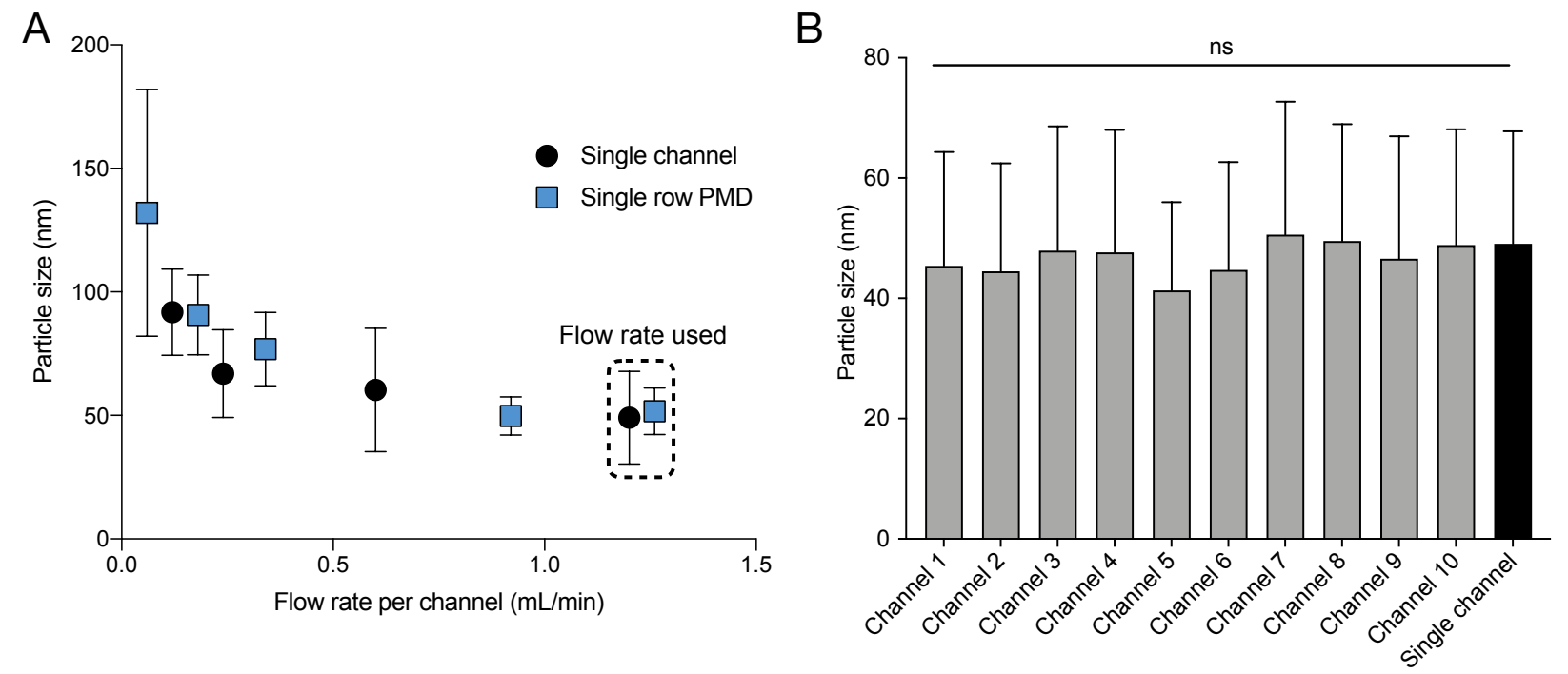

Figure S2. Characterization of single stranded DNA (ssDNA) LNPs formulated by the single row PMD indicates uniform performance for each mixing channel.

(A) ssDNA LNPs were formulated at a variety of flow rates per channel $(0.1-1.26 \mathrm{~mL} / \mathrm{min})$, where LNP size ( \pm standard deviation) was dependent on flow rate until the smallest size $(50 \mathrm{~nm})$ LNPs were formed. This experiment was repeated with a microfluidic single channel device for comparison. $n=3$. (B) ssDNA LNPs were formulated with the single row PMD with individual outputs, and hydrodynamic size $( \pm$ standard deviation) was quantified by dynamic light scattering. $n=3$. Samples were compared by one-way ANOVA. ns: $\mathrm{p}>0.05$. 

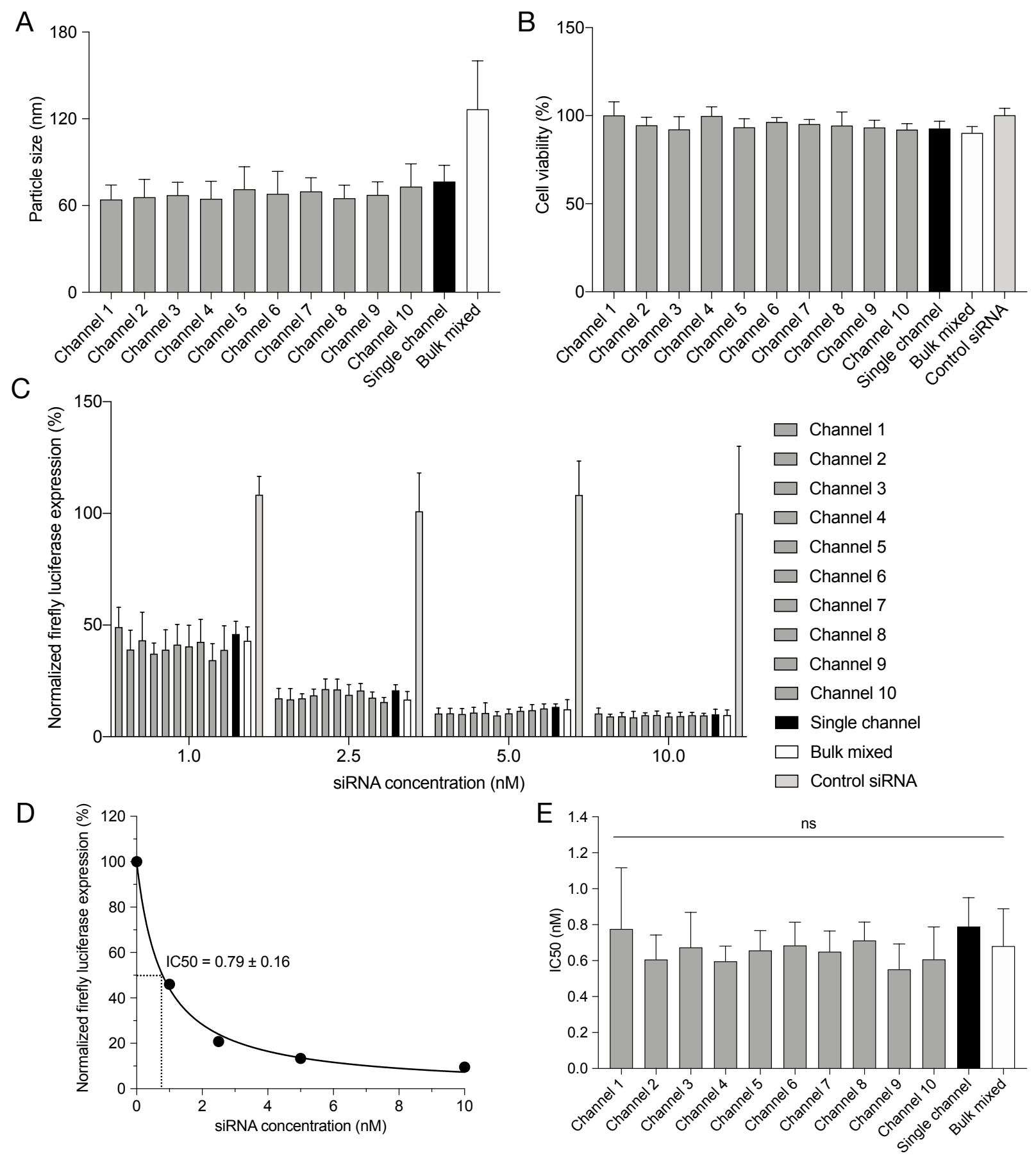

Figure S3. Luciferase siRNA LNPs were formulated by the single row PMD to confirm uniform LNP physical properties and potency across all 10 mixing channels.

LNP size, size distribution, knockdown efficiency in vitro, and cell viability were quantified for 1-10 nM siRNA doses in HeLa cells. (A) Particle size ( \pm standard deviation) was quantified for LNPs formulated by each channel of the PMD, microfluidic single channel device, or bulk mixing by dynamic light scattering. (B) Cell viability ( \pm standard deviation) was quantified by a CellTiter-Glo kit for cells treated with $10 \mathrm{nM}$ (max dose) firefly luciferase siRNA LNPs. Data is normalized to untreated cells with background subtracted. $\mathrm{n}=5$. (C) Dose response for HeLa cells treated with 1-10 nM firefly luciferase siRNA LNPs, showing luciferase expression ( \pm standard deviation) normalized to untreated cells with background 
subtracted. $\mathrm{n}=10$. (D) Example $\mathrm{IC}_{50}$ calculation, where luciferase expression is plotted versus siRNA concentration, and an exponential decay curve determines the siRNA concentration for $50 \%$ expression. (E) Calculated $\mathrm{IC}_{50}$ ( \pm standard deviation) from the data in $(\mathrm{C})$ for each channel and alternative formulation methods. Samples were compared by one-way ANOVA. ns: $p>0.05$. 
A

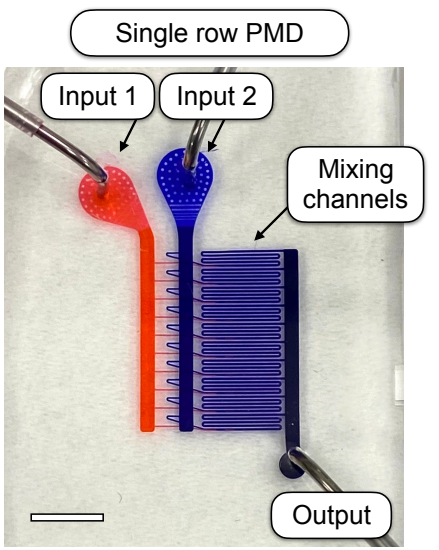

C
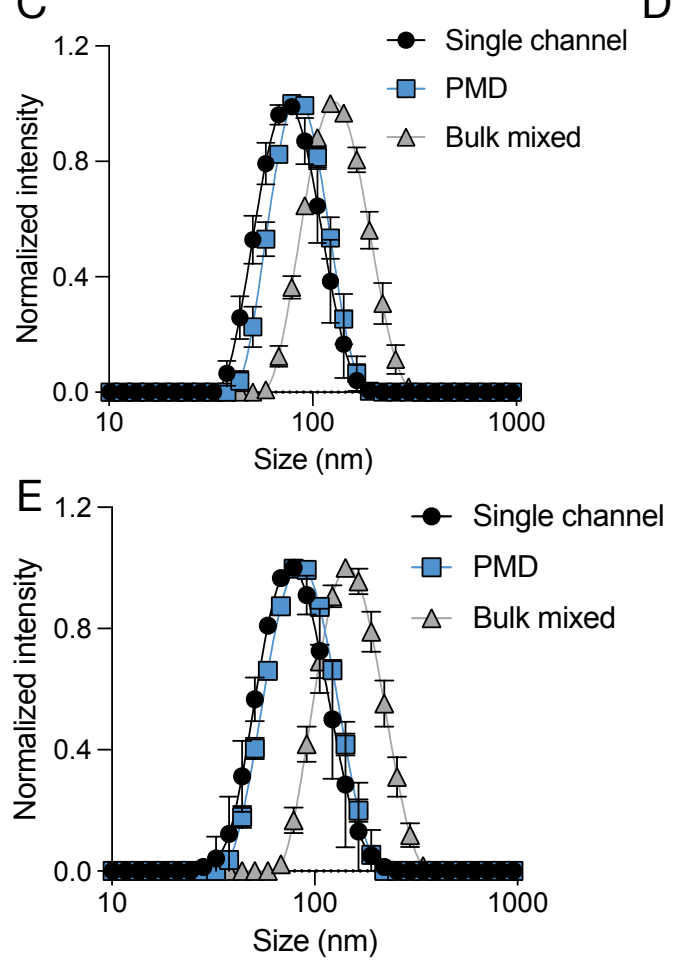

B

\begin{tabular}{|c|c|c|c|c|}
\hline LNP & $\begin{array}{c}\text { Diameter } \\
(\mathrm{nm})\end{array}$ & PDI & $\begin{array}{c}\text { FVII siRNA } \\
(\mathrm{ng} / \mathrm{uL})\end{array}$ & $\begin{array}{c}\text { Encapsulation } \\
\text { efficiency }(\%)\end{array}$ \\
\hline $\begin{array}{c}\text { Microfluidic } \\
\text { single } \\
\text { channel }\end{array}$ & $67.3 \pm 17.3$ & $0.068 \pm 0.017$ & $244.5 \pm 3.8$ & $95.0 \pm 0.2$ \\
\hline $\begin{array}{c}\text { Single row } \\
\text { PMD }\end{array}$ & $82.2 \pm 21.4$ & $0.066 \pm 0.018$ & $281.0 \pm 4.2$ & $95.4 \pm 0.5$ \\
\hline Bulk mixed & $124.4 \pm 35.8$ & $0.083 \pm 0.015$ & $332.4 \pm 4.6$ & $95.6 \pm 0.7$ \\
\hline
\end{tabular}

$\mathrm{D}$

\begin{tabular}{|c|c|c|c|c|}
\hline LNP & $\begin{array}{c}\text { Diameter } \\
(\mathrm{nm})\end{array}$ & PDI & $\begin{array}{c}\text { Luciferase } \\
\text { mRNA (ng/ } \\
\text { uL) }\end{array}$ & $\begin{array}{c}\text { Encapsulation } \\
\text { efficiency (\%) }\end{array}$ \\
\hline $\begin{array}{c}\text { Microfluidic } \\
\text { single } \\
\text { channel }\end{array}$ & $77.3 \pm 25.4$ & $0.108 \pm 0.065$ & $53.7 \pm 1.5$ & $83.5 \pm 1.6$ \\
\hline $\begin{array}{c}\text { Single row } \\
\text { PMD }\end{array}$ & $82.4 \pm 24.6$ & $0.089 \pm 0.040$ & $101.6 \pm 2.0$ & $81.1 \pm 7.3$ \\
\hline Bulk mixed & $145.1 \pm 42.4$ & $0.085 \pm 0.027$ & $79.8 \pm 1.1$ & $61.5 \pm 1.6$ \\
\hline
\end{tabular}

Figure S4. Microfluidic-formulated LNPs encapsulating Factor VII siRNA or luciferase mRNA had a small size and high encapsulation efficiency compared to bulk formulation.

(A) Photograph of the PMD with different colored dyes (blue, orange) for each inlet. Scale bar: $5 \mathrm{~mm}$. (B,D) Tables reporting the diameter (dynamic light scattering intensity-based z-average), polydispersity index (PDI), RNA concentration, and encapsulation efficiency ( \pm standard deviation) for each formulation of either Factor VII siRNA LNPs (B) or firefly luciferase mRNA LNPs (D). (C,E) Dynamic light scattering (DLS) curves showing the intensity distribution ( \pm standard deviation) of either Factor VII siRNA LNPs (C) or firefly luciferase mRNA LNPs (E) for the different formulation methods. 
A
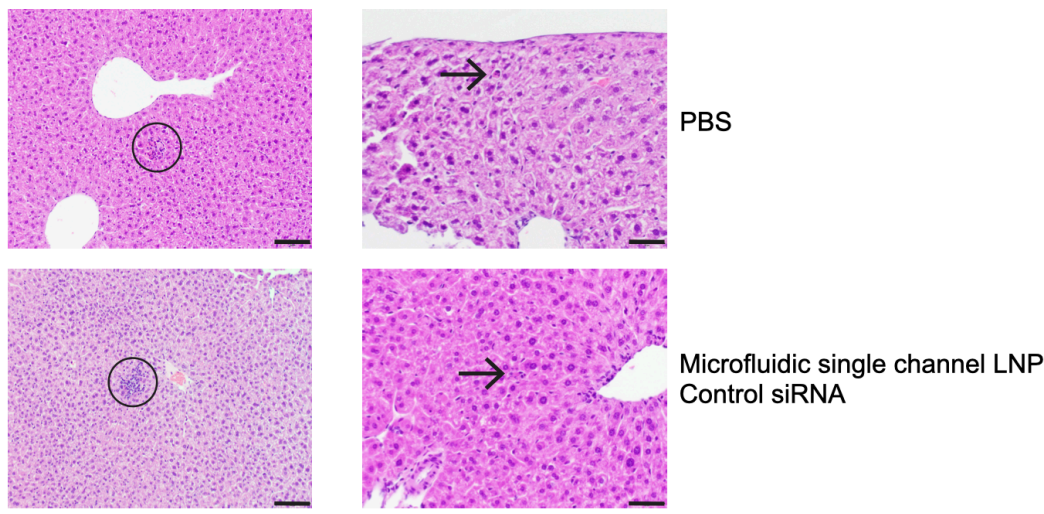

Control siRNA
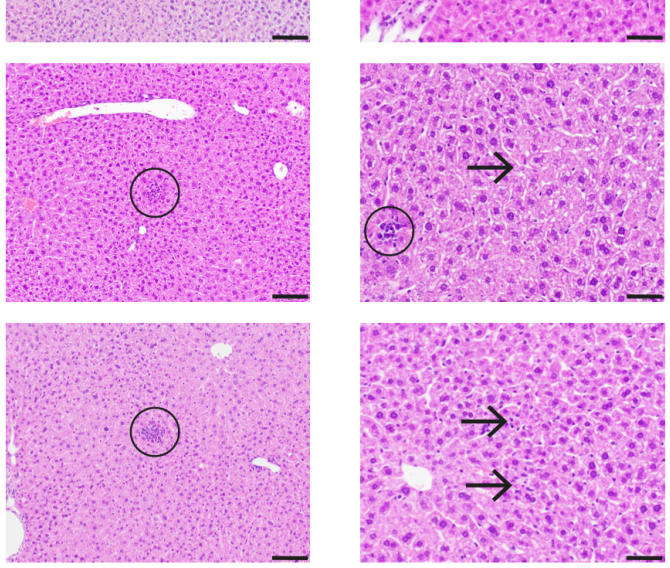

Microfluidic single channel LNP

Factor VII siRNA
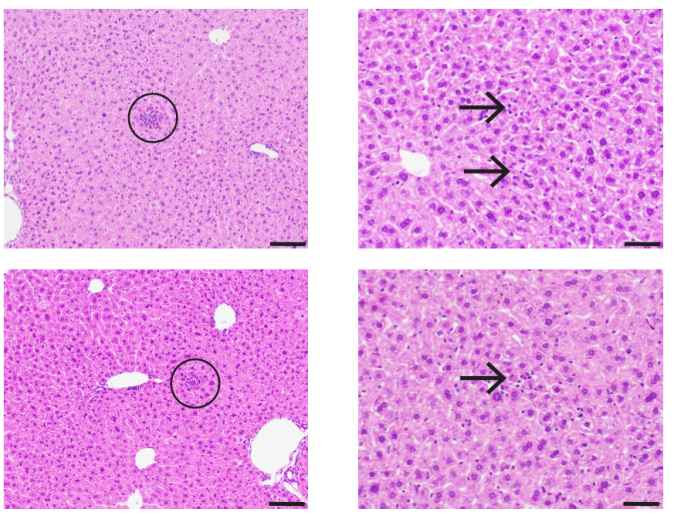

Single row PMD LNP

Factor VII siRNA

Figure S5. Mouse liver histopathology indicates no significant differences in toxicity between LNP formulations.

C57BL $/ 6$ mice were dosed with $0.2 \mathrm{mg} / \mathrm{kg}$ Factor VII siRNA LNPs, and liver samples were examined 48 hours after LNP administration. (A) Representative images of hematoxylin and eosin staining are shown for each experimental group at 20X (left) and 40X (right) magnification. Minor mixed lymphocytic infiltrates (circles) and individual necrotic hepatocytes (arrows) were observed in all groups and determined to be incidental and not secondary to test article. Scale bars: $100 \mu \mathrm{m}$ (left panel), $50 \mu \mathrm{m}$ (right panel). 
A

Reference range for AST: 32-122 IU/L

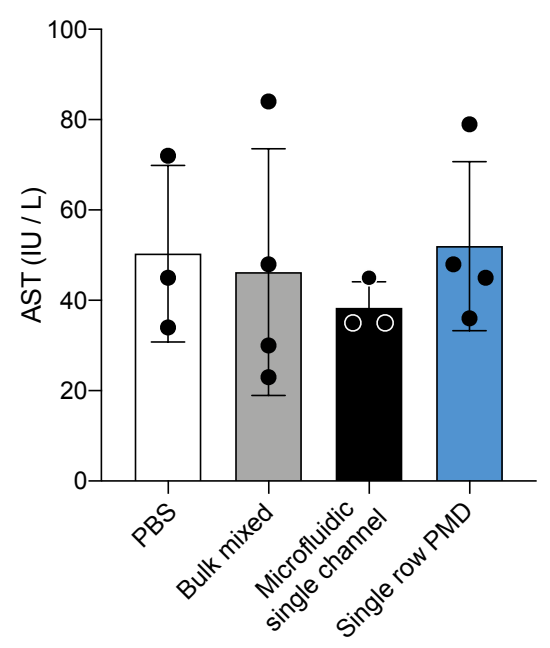

B

Reference range for ALT: 18-94 IU/L

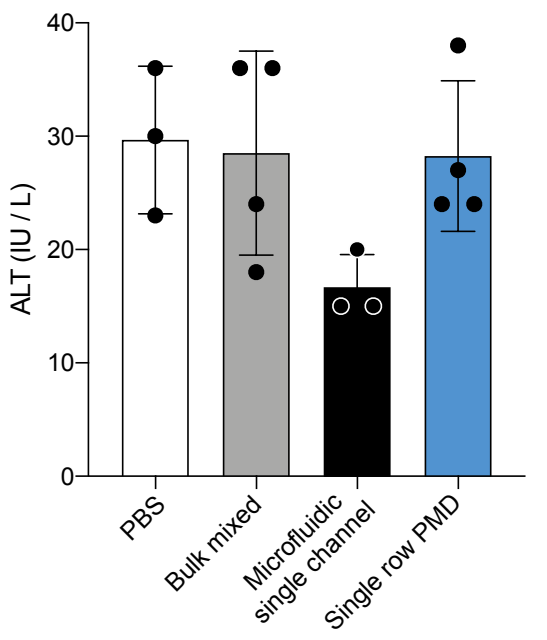

C

Reference range for ALP: 68-132 IU/L

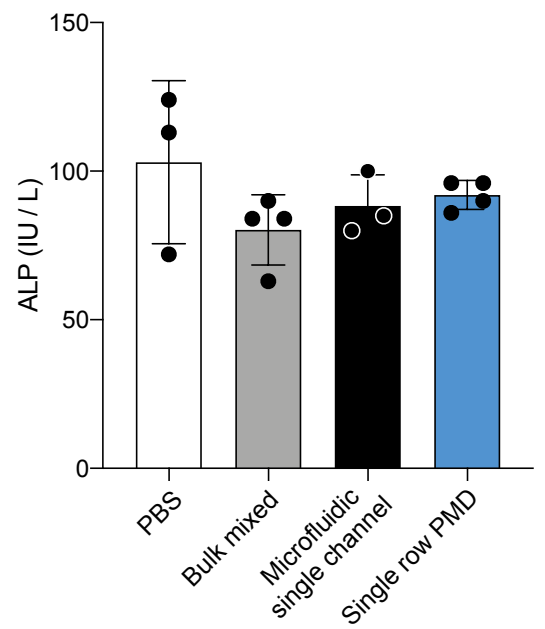

Figure S6. Liver toxicity assays indicate no clinically significant differences in toxicity between LNP formulations.

C57BL/6 mice were dosed with $0.2 \mathrm{mg} / \mathrm{kg}$ luciferase mRNA LNPs, and liver enzymes were quantified 4 hours after injection. (A) Aspartate transaminase (AST) quantification ( \pm standard deviation) for the formulation groups and controls. $n=3-4$. (B) Alanine transaminase (ALT) quantification $( \pm$ standard deviation) for the formulation groups and controls. $n=3-4$. (C) Alkaline phosphatase (ALP) quantification ( \pm standard deviation) for the formulation groups and controls. $n=3-4$. Reference ranges are listed for C57BL/6J mice for each enzyme (A-C). 


\section{References}

(1) Romanowsky, M. B.; Abate, A. R.; Rotem, A.; Holtze, C.; Weitz, D. A. High Throughput Production of Single Core Double Emulsions in a Parallelized Microfluidic Device. Lab Chip 2012, 12 (4), 802-807. https://doi.org/10.1039/c2lc21033a.

(2) Jeong, H. H.; Yelleswarapu, V. R.; Yadavali, S.; Issadore, D.; Lee, D. Kilo-Scale Droplet Generation in Three-Dimensional Monolithic Elastomer Device (3D MED). Lab Chip 2015, 15 (23), 4387-4392. https://doi.org/10.1039/c5lc01025j.

(3) Love, K. T.; Mahon, K. P.; Levins, C. G.; Whitehead, K. A.; Querbes, W.; Dorkin, J. R.; Qin, J.; Cantley, W.; Qin, L. L.; Racie, T.; Frank-Kamenetsky, M.; Yip, K. N.; Alvarez, R.; Sah, D. W. Y.; de Fougerolles, A.; Fitzgerald, K.; Koteliansky, V.; Akinc, A.; Langer, R.; Anderson, D. G. Lipid-like Materials for LowDose, in Vivo Gene Silencing. Proc. Natl. Acad. Sci. 2010, 107 (5), 1864-1869. https://doi.org/10.1073/pnas.0910603106.

(4) Billingsley, M. M.; Singh, N.; Ravikumar, P.; Zhang, R.; June, C. H.; Mitchell, M. J. Ionizable Lipid Nanoparticle-Mediated MRNA Delivery for Human CAR T Cell Engineering. Nano Lett. 2020, 20 (3), 1578-1589. https://doi.org/10.1021/acs.nanolett.9b04246.

(5) Pardi, N.; Tuyishime, S.; Muramatsu, H.; Kariko, K.; Mui, B. L.; Tam, Y. K.; Madden, T. D.; Hope, M. J.; Weissman, D. Expression Kinetics of Nucleoside-Modified MRNA Delivered in Lipid Nanoparticles to Mice by Various Routes. J. Control. Release 2015, 217, 345-351. https://doi.org/10.1016/j.jconrel.2015.08.007.

(6) Otto, G. P.; Rathkolb, B.; Oestereicher, M. A.; Lengger, C. J.; Moerth, C.; Micklich, K.; Fuchs, H.; Gailusdurner, V.; Wolf, E.; de Angelis, M. H. Clinical Chemistry Reference Intervals for C57BL/6J, C57BL/6N, and $\mathrm{C} 3 \mathrm{HeB} / \mathrm{FeJ}$ Mice (Mus Musculus). J. Am. Assoc. Lab. Anim. Sci. 2016, 55 (4), 375-386. 